\title{
ARTICLE OPEN \\ High-performance bifunctional polarization switch chiral metamaterials by inverse design method
}

\author{
Chuanbao Liu ${ }^{1,2,3}$, Yang Bai ${ }^{1,2}$, Ji Zhou $^{3}$, Qian Zhao ${ }^{4}$, Yihao Yang ${ }^{5}$, Hongsheng Chen ${ }^{5}$ and Lijie Qiao ${ }^{1,2}$
}

Multifunctional polarization controlling plays an important role in modern photonics, but their designs toward broad bandwidths and high efficiencies are still rather challenging. Here, by applying the inverse design method of model-based theoretical paradigm, we design cascaded chiral metamaterials for different polarization controls in oppositely propagating directions and demonstrate their broadband and high-efficiency performance theoretically and experimentally. Started with the derivation of scattering matrix towards specified polarization control, a chiral metamaterial is designed as a meta-quarter-wave plate for the forward propagating linearly polarized wave, which converts the $x$ - or $y$-polarized wave into a nearly perfect left- or right-handed circularly polarized wave; intriguingly, it also serves as a $45^{\circ}$ polarization rotator for the backward propagating linearly polarized waves. This bifunctional metamaterial shows a high transmission as well as a broad bandwidth due to the Fabry-Perot-like interference effect. Using the similar approach, an abnormal broadband meta-quarter-wave plate is achieved to convert the forward $x$ - and $y$-polarized or the backward $y$ - and $x$-polarized waves into left- and right-handed circularly polarized waves with high transmission efficiencies. The integration of multiple functions in a single structure endows the cascaded chiral metamaterials with great interests for the high-efficiency polarization-controlled applications.

npj Computational Materials (2019)5:93

; https://doi.org/10.1038/s41524-019-0230-z

\section{INTRODUCTION}

The field of materials science has evolved from empirical paradigm to theoretical, computational, and data-driven science. ${ }^{1}$ Traditional material design always follows a route from a certain material with specific structure to the final properties, i.e., forward material design, but such a trial-and-error process takes great pains for time and effort to obtain a material with desired properties. With the rapid development of theoretical, computational, and data-driven paradigms recently, the inverse material design, starting from a specific function to the corresponding structure, opens up new avenues for accelerated materials discovery and application. Compared with natural materials, metamaterials constructed by artificial meta-atoms have much higher designability to facilitate an inverse design approach, so that the unavailable properties by traditional approach can be well achieved.

The emergence of metamaterials breaks the natural materials' constraint and brings an unprecedented opportunity to fully control the electromagnetic waves (EMWs) from microwave to optical frequencies, including polarization, phase, and amplitude. ${ }^{2-5}$ Polarization, a basic characteristic of EMW, conveys valuable information and serves as a cornerstone in many optical phenomena. Manipulating the polarization of EMWs is vital to numerous applications from signal communication, spectroscopy to microscopy. However, traditional components suffer from bulky size and limited material resources. Chiral metamaterials are an important subset of metamaterials and have an additional mirror symmetry breaking in the propagation direction or two- dimensional plane. They can arbitrarily modulate the polarization of EMWs at sub-wavelength scales due to the strong chiral response from the handedness-selective EM resonance in the structures, demonstrating many intriguing phenomena and applications, such as negative refractive index, ${ }^{6,7}$ circular dichroism, ${ }^{8-10}$ asymmetric transmission, ${ }^{11-15}$ polarization rotation and conversion, ${ }^{16-21}$ chiral-selective absorber, ${ }^{22,23}$ and so forth. Recently, multifunctional metamaterials are highly desired to meet the increasing demand for miniaturization and complex application scenarios. Some researchers design anisotropic or active metamaterials to achieve the functionality integration by changing the helicity, ${ }^{24-27}$ polarization, ${ }^{28-30}$ propagation direction of incident waves, ${ }^{31}$ and external stimulation. ${ }^{32-34}$ All these studies aim at reshaping wavefronts, but more important and fundamental manipulation of polarization, such as basic functionalities of linear polarization rotation, linear-to-circular (or vice versa) polarization conversion, are rarely involved.

Most chiral metamaterials for polarization control are designed with a specified function. Typically, bilayer chiral metamaterials, consisting of two-layer closely stacked and mutually twisted resonators, are investigated for polarization rotation with subwavelength thicknesses; ${ }^{16}$ multilayer gratings ${ }^{17}$ and gold helix structures $^{18}$ are proposed for broadband linear-to-circular and circular polarization conversion. Previous tunable or reconfigurable multifunctional chiral metamaterials ${ }^{35-39}$ usually need additional control systems, resulting in the difficulties in fabrication, reliability, and signal repeatability. Although the integration of circular asymmetric transmission with wavefront manipulation can

\footnotetext{
${ }^{1}$ Beijing Advanced Innovation Center for Materials Genome Engineering, University of Science and Technology Beijing, Beijing 100083 , China; ${ }^{2}$ Key Laboratory of Environmental Fracture (Ministry of Education), Institute for Advanced Materials and Technology, University of Science and Technology Beijing, Beijing 100083, China; ${ }^{3}$ State Key Laboratory of New Ceramics and Fine Processing, Tsinghua University, Beijing 100084, China; ${ }^{4}$ State Kay Laboratory of Tribology, Department of Mechanical Engineering, Tsinghua University, Beijing 100084, China and ${ }^{5}$ State Key Laboratory for Modern Optical Instrumentation, Zhejiang University, Hangzhou 310027, China Correspondence: Yang Bai (baiy@mater.ustb.edu.cn)
}

Received: 19 June 2019 Accepted: 27 August 2019

Published online: 17 September 2019 


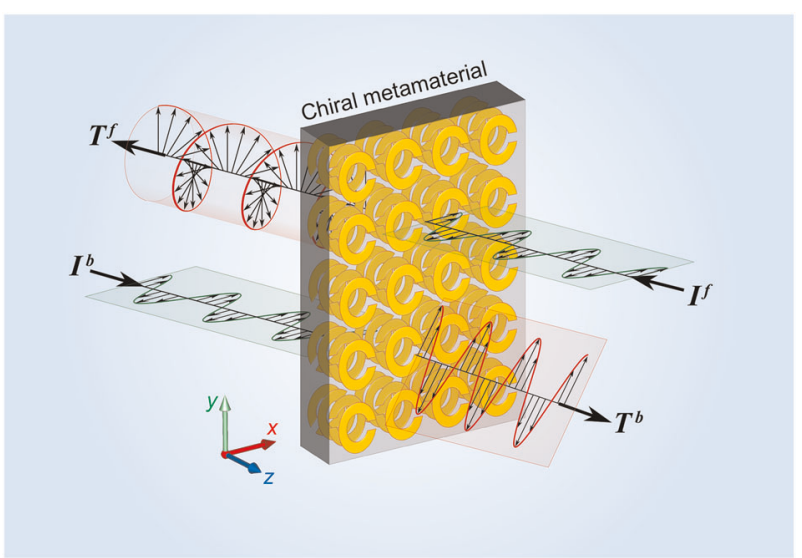

Fig. 1 Schematic of a high-performance bifunctional chiral metamaterial which behaves as an $\mathrm{m}$-QWP to convert the forward propagating LP wave into CP wave; while it acts as a $45^{\circ}$ polarization rotator for the backward propagating LP wave

make chiral metamaterials more versatile, the involved polarization control only relates to the circular polarization conversion. ${ }^{14,40}$ Recently, Yang et al. design a direction-controlled bifunctional metasurface polarizer, but it suffers from a low transmission and narrow bandwidth due to the strong coupling. ${ }^{41}$ Despite of the high importance in applications, it remains elusive to achieve a passive metamaterial for multifunctional polarization control with a high efficiency and broad bandwidth.

Aiming at such difficulty in multifunctional metamaterials, we utilize the inverse design method of model-based theoretical paradigm, which not only facilitates to discover metamaterials with desired functional characteristics but also endows people with clear physical understanding. Based on the scattering matrix, we obtain the restrictive conditions for specified polarization control and propose two multifunctional metamaterials with simple cascade configurations as examples. One exhibits linear-tocircular polarization conversion (meta-quarter-wave plate, mQWP) and $45^{\circ}$ polarization rotation (polarization rotator) for the forward and backward propagating linearly polarized (LP) waves, respectively, as illustrated in Fig. 1; while the other converts the forward and backward propagating LP waves into a circularly polarized (CP) wave with different handedness (an abnormal mQWP). Theoretical calculation, numerical simulation, and experimental results all demonstrated that the chiral metamaterials both have an excellent waveform output and an ultrahigh transmission within a broad bandwidth. The obtained multifunction and excellent properties will greatly enrich the device design and be highly desired by the practical applications.

\section{RESULTS}

Theoretical analysis

In order to understand the mechanism of polarization control, we used the scattering matrix $\boldsymbol{S}$ to describe the scattering properties of metamaterials where only the zero-order Bloch mode exists. Because of the involved polarization rotation, it is convenient to use an equivalent four-port (two-port with $x, y$-polarization) network in the circuit theory to tackle this situation. Especially, when the media at the both sides of a metamaterial are same, $S$ parameters are equivalent to transmission and reflection coefficients. The scattering matrix $S$ relates to the outgoing $\left(\overleftarrow{E_{x}^{o}}, \overparen{E_{y}^{\circ}}, \overrightarrow{E_{x}^{o}}\right.$ and $\left.\overrightarrow{E_{y}^{O}}\right)$ and incoming electric fields $\left(E_{x}^{i}, \overrightarrow{E_{y}^{i}}, E_{x}^{i}\right.$ and $\left.E_{y}^{i}\right)$, and can be written as

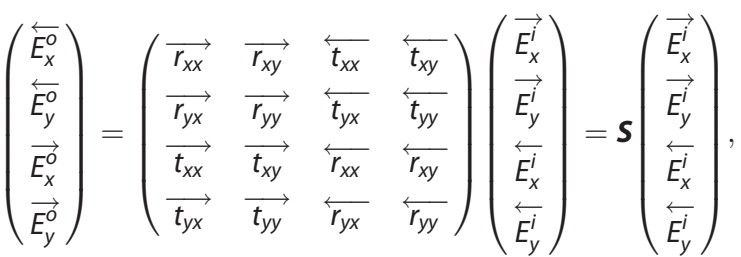

where the symbols $\rightarrow$ and $\leftarrow$ represent the forward and backward propagations, respectively, the elements $r_{i j}$ and $t_{i j}$ are the reflection and transmission coefficients of the $i$-polarization for a $j$-polarized incident wave $(i, j=x, y)$. If the metamaterial is lossless, the real part of a complex power flow equals zero, leading to a unitary scattering matrix $\boldsymbol{S}$ which satisfies the following relation

$\boldsymbol{S}^{\dagger} \mathbf{S}=\boldsymbol{I}$

where $\boldsymbol{S}^{\dagger}$ denotes the conjugate transpose, $\boldsymbol{I}$ is the identity matrix. For an ideal unreflective metamaterial, the $\boldsymbol{S}$ matrix can be expressed as

$\mathbf{S}=\left(\begin{array}{cccc}0 & 0 & \overleftarrow{t_{x x}} & \overleftarrow{t_{x y}} \\ 0 & 0 & \overleftarrow{t_{y x}} & \overleftarrow{t_{y y}} \\ \underset{t_{x x}}{\stackrel{t_{y x}}{\longrightarrow}} & \stackrel{t_{x y}}{\longrightarrow} & 0 & 0 \\ \longrightarrow & 0 & 0\end{array}\right)$

In the absence of Faraday media, the reciprocity theorem is applied, where $S$-parameters equal to their transposes $S_{m n}=S_{n m}$ $(m, n=1,2,3,4)$. By inserting Eq. (3) into Eq. (2), we can get the restrictive conditions for the forward propagating transmission matrix

$\boldsymbol{T}^{f}=\left(\begin{array}{ll}\overrightarrow{t_{x x}} & \overrightarrow{t_{x y}} \\ \underset{t_{y x}}{\longrightarrow} & \overrightarrow{t_{y y}}\end{array}\right)=\left(\begin{array}{cc}\sin \phi e^{i \xi_{x x}} & \cos \phi e^{i \xi_{x y}} \\ \cos \phi e^{i \xi_{y x}} & \sin \phi e^{i \xi_{y y}}\end{array}\right)$,

where $\sin \phi$ and $\cos \phi\left(0^{\circ} \leq \phi \leq 90^{\circ}\right)$ are the transmission amplitudes; $\xi_{x x}, \xi_{y x}, \xi_{x y}$, and $\bar{\xi}_{y y}$ are transmission phases of four components. Especially when $0^{\circ}<\phi<90^{\circ}, \xi=\xi_{y y}+\xi_{x x}-\xi_{y x}$ $-\xi_{x y}=(2 n+1) \pi$, where $n=0, \pm 1, \pm 2 \ldots$ is an integer. Let us consider an ideal unreflective and lossless $\mathrm{m}$-QWP which converts the forward propagating $x$-polarized wave into LCP wave, the $\boldsymbol{T}$ matrix can be written as

$\boldsymbol{T}^{f}=\left(\begin{array}{cc}\frac{1}{\sqrt{2}} & \overrightarrow{t_{x y}} \\ -\frac{i}{\sqrt{2}} & \overrightarrow{t_{y y}}\end{array}\right)$.

Comparing Eq. (5) with Eq. (4), we can get

$\boldsymbol{T}^{f}=\frac{1}{\sqrt{2}}\left(\begin{array}{cc}1 & e^{i \theta} \\ -i & e^{i(\theta+\pi / 2)}\end{array}\right)$.

Obviously, structures with such $\boldsymbol{T}$ matrix (Eq. (6)) can convert the forward propagating $x$ - or $y$-polarized wave into LCP or RCP wave, regardless of the value of $\theta$ (an arbitrary real number). However, the choice of variable $\theta$ will significantly affects the transmission performance for the backward propagating LP waves. Due to the absence of Faraday media, the $\boldsymbol{T}^{b}$ matrix for the backward propagating wave is obtained by simply interchanging the offdiagonal elements in the forward $\boldsymbol{T}^{f}$ matrix and written as

$\boldsymbol{T}^{b}=\frac{1}{\sqrt{2}}\left(\begin{array}{cc}1 & -i \\ e^{i \theta} & e^{i(\theta+\pi / 2)}\end{array}\right)$.

It is noted that the relationship between $T^{f}$ and $T^{b}$ is only valid for a particular base where the Cartesian coordinate system is kept unchanged. From Eq. (7), the proper selection of $\theta$ can realize linear-to-circular polarization conversion or linear polarization rotation for the backward propagating LP waves. In the following 
section, we first choose several typical values of $\theta$ to explore abundant functionalities of polarization control. Then, we demonstrate how to construct these structures with peculiar $\boldsymbol{T}$ matrices.

For example, when the variable $\theta=-\pi / 2$, the forward and backward propagating matrices are expressed as

$$
\boldsymbol{T}_{\theta=-\pi / 2}^{f}=\boldsymbol{T}_{\theta=-\pi / 2}^{b}=\frac{1}{\sqrt{2}}\left(\begin{array}{cc}
1 & -i \\
-i & 1
\end{array}\right) \text {. }
$$

Equation (8) indicates that this kind of structure behaves as a traditional $\mathrm{m}$-QWP of converting $x$ - or $y$-polarized wave into LCP or RCP waves, regardless of propagation directions. Meanwhile, the characteristic of Eq. (8) implies the elements are not independent but connected by trigonometric functions, a rotation by an angle $\varphi=45^{\circ}$ leads to a diagonal form

$\boldsymbol{T}_{\text {new }}^{f}=\boldsymbol{D}_{\varphi} \boldsymbol{T}_{\theta=-\pi / 2}^{f} \boldsymbol{D}_{\varphi}^{-1}=\frac{1}{\sqrt{2}}\left(\begin{array}{cc}1+i & 0 \\ 0 & 1-i\end{array}\right)$,

where $\boldsymbol{D}_{\varphi}=\left(\begin{array}{cc}\cos \varphi & -\sin \varphi \\ \sin \varphi & \cos \varphi\end{array}\right)$ is the rotation matrix. Hence, this structure can be regarded as an m-QWP with its slow axis at $45^{\circ}$ with respect to both the $x$ and $y$ axes.

For $\theta=0$, the forward and backward propagating matrices become

$$
\begin{aligned}
& \boldsymbol{T}_{\theta=0}^{f}=\frac{1}{\sqrt{2}}\left(\begin{array}{cc}
1 & 1 \\
-i & i
\end{array}\right), \\
& \boldsymbol{T}_{\theta=0}^{b}=\frac{1}{\sqrt{2}}\left(\begin{array}{cc}
1 & -i \\
1 & i
\end{array}\right) .
\end{aligned}
$$

From Eqs. (10) and (11), we conclude that this kind of structure has two different functions. For the forward LP waves, this structure behaves as an $\mathrm{m}$-QWP to realize linear-to-circular polarization conversion; while it acts as a $45^{\circ}$ polarization rotator for the backward propagating LP waves. To construct this bifunctional structure, we decompose the Eq. (10) into two matrices

$$
\boldsymbol{T}_{\theta=0}^{f}=\frac{1}{\sqrt{2}}\left(\begin{array}{cc}
1 & -i \\
-i & 1
\end{array}\right)\left(\begin{array}{ll}
1 & 0 \\
0 & i
\end{array}\right) \text {. }
$$

According to the transfer matrix method, an LP wave passes through two structures ( 1 and 2 ) successively, the overall $\boldsymbol{T}_{t}$ matrix can be written as

$\boldsymbol{T}_{t}=\boldsymbol{T}_{2} \cdot \boldsymbol{P} \cdot \boldsymbol{T}_{1}$,

where $\boldsymbol{P}=\operatorname{diag}\left(e^{-i n k_{0} d}, e^{-i n k_{0} d}\right)$ is a phase delay term caused by the EMW's propagation in homogenous medium (refractive index $n$, thickness $d$, and vacuum wave number $k_{0}$ ) between two structures. For convenience, we neglect the phase delay term here which has no influence on the polarization control. Hence, this kind of bifunctional structure can be regarded as a cascading of two m-QWPs with a twist angle of $45^{\circ}$, where the slow axis of the front $\mathrm{m}-\mathrm{QWP}$ is along with $x$ axis, as shown in Fig. 1.

For $\theta=\pi / 2$, we can get the forward and backward propagating matrices

$$
\begin{aligned}
& \boldsymbol{T}_{\theta=\pi / 2}^{f}=\frac{1}{\sqrt{2}}\left(\begin{array}{cc}
1 & i \\
-i & -1
\end{array}\right), \\
& \boldsymbol{T}_{\theta=\pi / 2}^{b}=\frac{1}{\sqrt{2}}\left(\begin{array}{cc}
1 & -i \\
i & -1
\end{array}\right) .
\end{aligned}
$$

The Eqs. (14) and (15) indicate structures having such properties can convert the forward $x$-polarized or $y$-polarized wave into LCP or RCP wave; while it realizes an inverse function of converting the backward $x$-polarized or $y$-polarized wave into RCP or LCP wave.
Similarly, we decompose the Eq. (14) into two matrices

$\boldsymbol{T}_{\theta=\pi / 2}^{f}=\frac{1}{\sqrt{2}}\left(\begin{array}{cc}1 & -i \\ -i & 1\end{array}\right)\left(\begin{array}{cc}1 & 0 \\ 0 & -1\end{array}\right)$.

According to Eq. (16), this abnormal m-QWP can be obtained by cascading a meta-half-wave plate ( $\mathrm{m}$-HWP) and an $\mathrm{m}$-QWP with a twist angle of $45^{\circ}$, where the slow axis of the front $\mathrm{m}-\mathrm{HWP}$ is along with $x$ axis. Besides, the m-HWP can also be decomposed by a cascaded $\mathrm{m}$-QWP pair with a twist angle of $0^{\circ}$ or $180^{\circ}$. Therefore, this abnormal $\mathrm{m}$-QWP is entirely made up of the traditional $\mathrm{m}$ QWPs.

It's noted that the construction of bifunctional chiral metamaterial or abnormal $\mathrm{m}$-QWP by cascading the traditional $\mathrm{m}$-QWPs can be also guided and optimized by tensor sheet admittances (see Supplementary Information, Section I). ${ }^{19,20}$ Furthermore, if loss and reflection are taken into consideration in scattering matrices, the reciprocity is kept while the unitary property is broken, so that the components of transmission matrix (Eq. (4)) can be tuned independently, complicating the theoretical analysis. In the following section, we first design a high-performance $\mathrm{m}$ QWP with a high transmission in broadband, and then combine $m$-QWPs with a twist angle to realize the diverse polarization control.

Meta-quarter-wave plate $(\theta=-\pi / 2)$

To construct high-performance multifunctional chiral metamaterials, we design an m-QWP to convert the LP wave into CP wave with a high transmission in a broad bandwidth. Single-layer metasurface can be served as an $\mathrm{m}-\mathrm{QWP}$, but the large dispersion limits its operation bandwidth due to the isolated electric response. An alternative way is using multilayer structure, where the formation of Fabry-Perot-like (F-P-like) interference between adjacent metasurfaces facilitates to achieve broadband and nondispersive polarization manipulation with high transmission. Figure $2 a$ depicts the optimized unit cell structure, composed of two metallic split ring resonators (SRRs) on both sides of an F4B board. We study the transmission properties of the two-layer metamaterial (TLM, structure 1) by using the commercial software CST Microwave Studio. Figure $2 \mathrm{~b}$ shows the simulated results for the normal incidence of LP waves. Because the TLM is mirrorsymmetric with respect to the $x z$ plane, the cross-polarization transmission coefficients are equal to zero. The amplitudes of co-polarization are greater than 0.8 and the phase difference of $\angle t_{y y}-\angle t_{x x}$ is about $90^{\circ} \pm 10^{\circ}$ (Fig. 2c) in the off-resonance frequency range of $10.5-12.5 \mathrm{GHz}$ (brown region). ${ }^{36}$ To quantitatively evaluate the performance of TLM as an m-QWP, we introduce Stokes parameters as

$$
\begin{gathered}
S_{0}=\left|t_{x}\right|^{2}+\left|t_{y}\right|^{2} \\
S_{1}=\left|t_{x}\right|^{2}-\left|t_{y}\right|^{2} \\
S_{2}=2\left|t_{x}\right|\left|t_{y}\right| \cos \Delta \varphi \\
S_{3}=2\left|t_{x}\right|\left|t_{y}\right| \sin \Delta \varphi,
\end{gathered}
$$

where $\left|t_{x}\right|$ and $\left|t_{y}\right|$ are the amplitude of transmitted electric fields along $x$ and $y$ directions, and $\Delta \varphi=\angle t_{y}-\angle t_{x}$ is the transmission phase difference. The ellipticity angle $x$ is defined as

$x=\frac{1}{2} \arcsin \left(S_{3} / S_{0}\right)$.

When the ellipticity angle $X= \pm 45^{\circ}$ or $0^{\circ}$, the transmitted waves are perfect RCP, LCP or LP waves, respectively. According to the simulated data, we obtain the ellipticity angle of $x \geq 40^{\circ}$ (Fig. $2 \mathrm{~d}$ ) in the frequency range of $10.5-12.5 \mathrm{GHz}$, i.e. a nearly perfect RCP wave output with high transmission efficiencies in broadband for an LP incident wave at $45^{\circ}$ with respect to the $x$ and $y$ axes. 
a

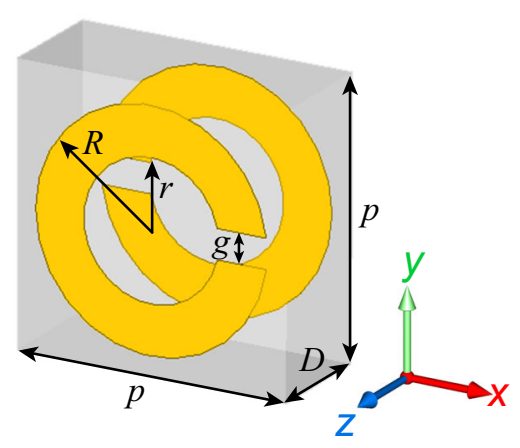

C

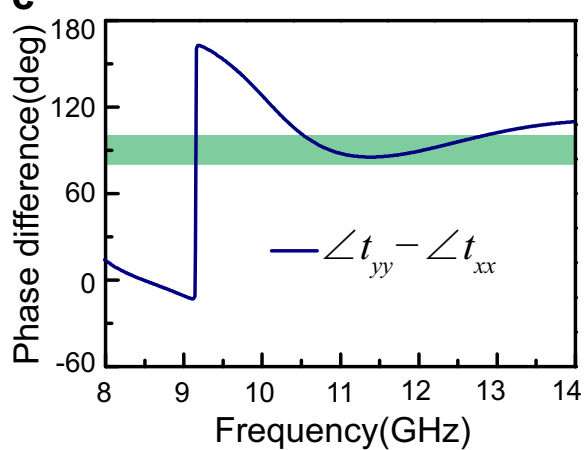

b

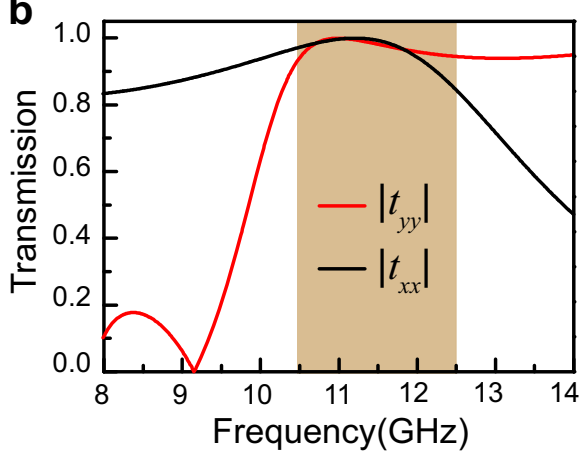

d

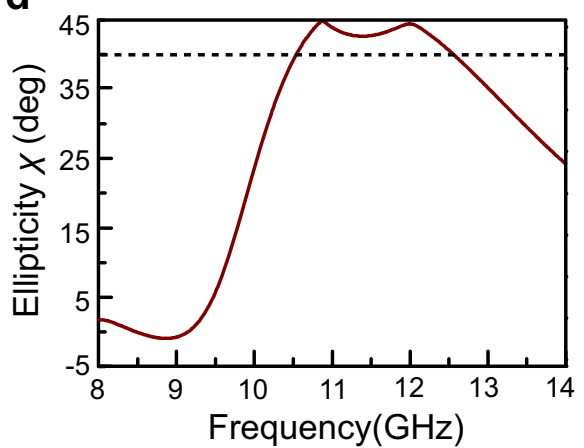

Fig. 2 TLM as an m-QWP. a Schematic and geometric dimensions of the unit cell: $p=7.0 \mathrm{~mm}, D=3.5 \mathrm{~mm}, r=1.75 \mathrm{~mm}, R=3.0 \mathrm{~mm}$, and $g=$ $0.75 \mathrm{~mm}$. b Simulated transmission spectra and $\mathbf{c}$ phase difference for $x$ - and $y$-polarized incident waves. $\mathbf{d}$ Calculated ellipticity angle $X$

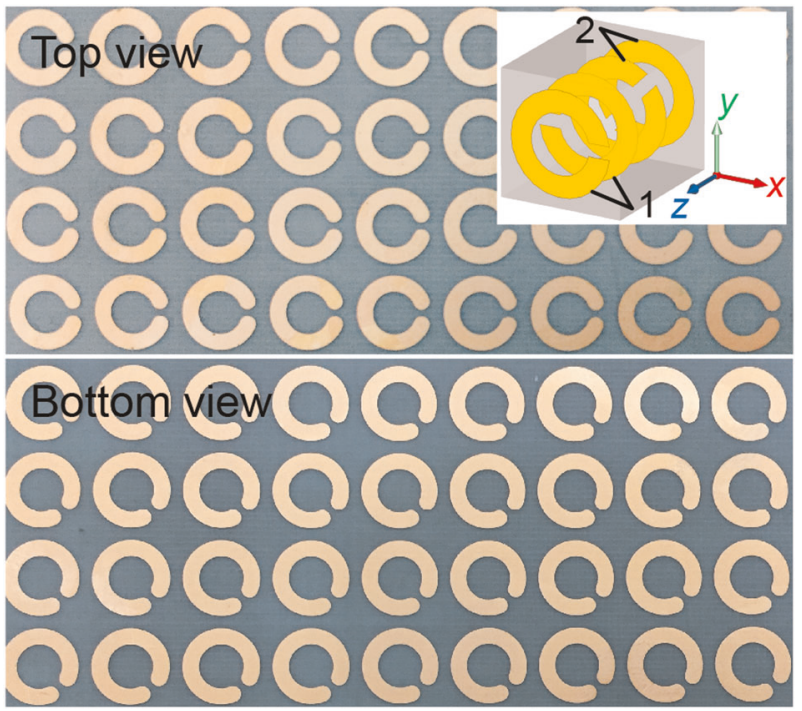

Fig. 3 Top and bottom views of FLCM as a bifunctional polarization controller. The inset shows the scheme of the unit cell

Bifunctional chiral metamaterial $(\theta=0)$

Next, we combine two TLMs (structure 1 and 2, Eq. (12)) together which are oriented at a twist angle of $45^{\circ}$ to each other and separated by an identical dielectric broad, resulting in a four-layer chiral metamaterial (FLCM), as shown in Fig. 3. Considering the coupling of two TLMs, we slightly adjust the periodicity of FLCM to $p=7.25 \mathrm{~mm}$ and keep other geometric parameters fixed. Numerical simulations and experiments are both carried out to study the electromagnetic properties of FLCM.
Figure $4 a$ shows the simulated transmission results for the forward propagating LP waves. For the incident wave polarized in the $x$ direction, the amplitudes of co-polarization transmission $\left|\overrightarrow{t_{x x}}\right|$ and cross-polarization transmission $\left|\overrightarrow{t_{y x}}\right|$ are closed to 0.7 in the off-resonance frequency range of $10.3-12.2 \mathrm{GHz}$ (brown region), indicating a high transmission efficiency where the total transmission defined as $\overrightarrow{T_{x}}=\left|\overrightarrow{t_{y x}}\right|^{2}+\left|\overrightarrow{t_{x x}}\right|^{2}$ is greater 0.85 , as shown in Fig. 4a1. Besides, the transmission phase difference of $\Delta \overrightarrow{\varphi_{x}}=\left\langle\overrightarrow{t_{y x}}-\overrightarrow{t_{x x}}\right.$ is about $-90^{\circ} \pm 10^{\circ}$ in the off-resonance region (Fig. 4a2, a5). Based on Eqs. (17) and (18), we calculate the ellipticity angle as shown in Fig. 4a6, where a nearly perfect LCP wave $\left(x \leq-40^{\circ}\right)$ with a high transmission is outputted in the frequency of $10.3-12.2 \mathrm{GHz}$. Similarly, the co-polarization transmission $\left|\overrightarrow{t_{y y}}\right|$ and the cross-polarization transmission $\left|\overrightarrow{t_{x y}}\right|$ are around 0.7 (Fig. 4a3), while the transmission phase difference of $\Delta \varphi_{y}=\left\langle\overrightarrow{t_{y y}}-\left\langle\overrightarrow{t_{x y}}\right.\right.$ is about $90^{\circ} \pm 10^{\circ}$ (Fig. $4 \mathrm{a} 4$, a5) for a normally incident $y$-polarized wave in the off-resonance frequency range of 10.0-12.5 GHz (brown region). The total transmission of $\overrightarrow{T_{y}}=$ $\left|\overrightarrow{t_{x y}}\right|^{2}+\left|\overrightarrow{t_{y y}}\right|^{2}$ is greater than 0.71 and reaches a maximum of 0.97 at $11.5 \mathrm{GHz}$. Figure $4 a 6$ shows the calculated ellipticity angle $X$ which is greater than $40^{\circ}$ in the off-resonance region, indicating a high transmission for the nearly perfect RCP wave output. Figure $4 \mathrm{~b}$ is the experimental results for the forward propagating LP waves, which agree with the simulated results well (Fig. 4a).

According to the reciprocity theorem, the transmission coefficients of cross-polarization interchange when the propagation direction is reversed; however, the transmission coefficients of copolarization keep unchanged. Hence, we can get the transmission phase difference for $x$ - and $y$-polarized incident waves in the 

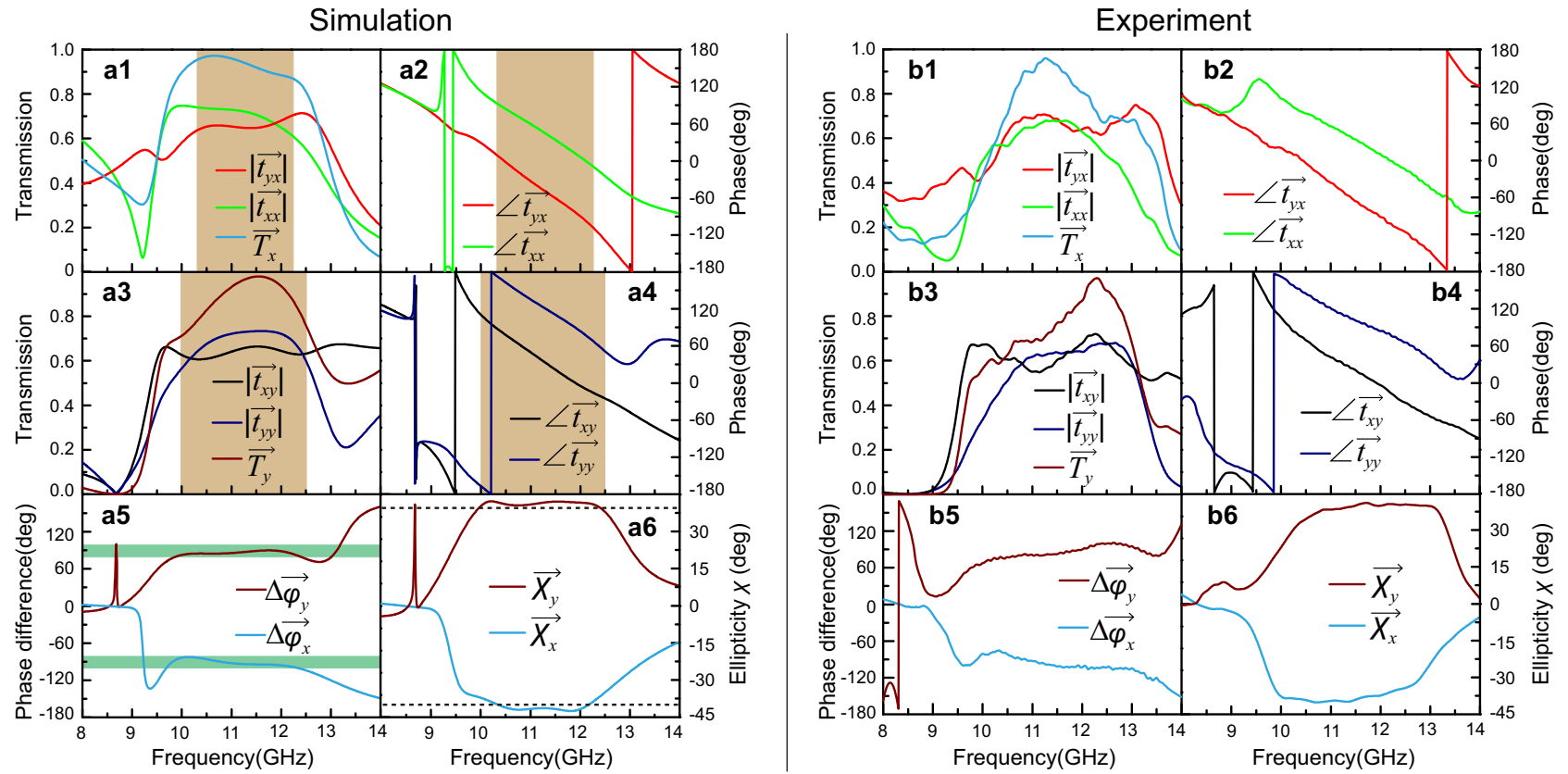

Fig. 4 a Simulated and $\mathbf{b}$ measured results of the FLCM for the forward propagating $x$ - and $y$-polarized waves. (a1, a3, b1 and b3) Transmission spectra, (a2, a4, b2 and b4) transmission phases, (a5 and b5) transmission phase differences, and (a6 and b6) calculated ellipticity angles $X$ of the transmitted waves
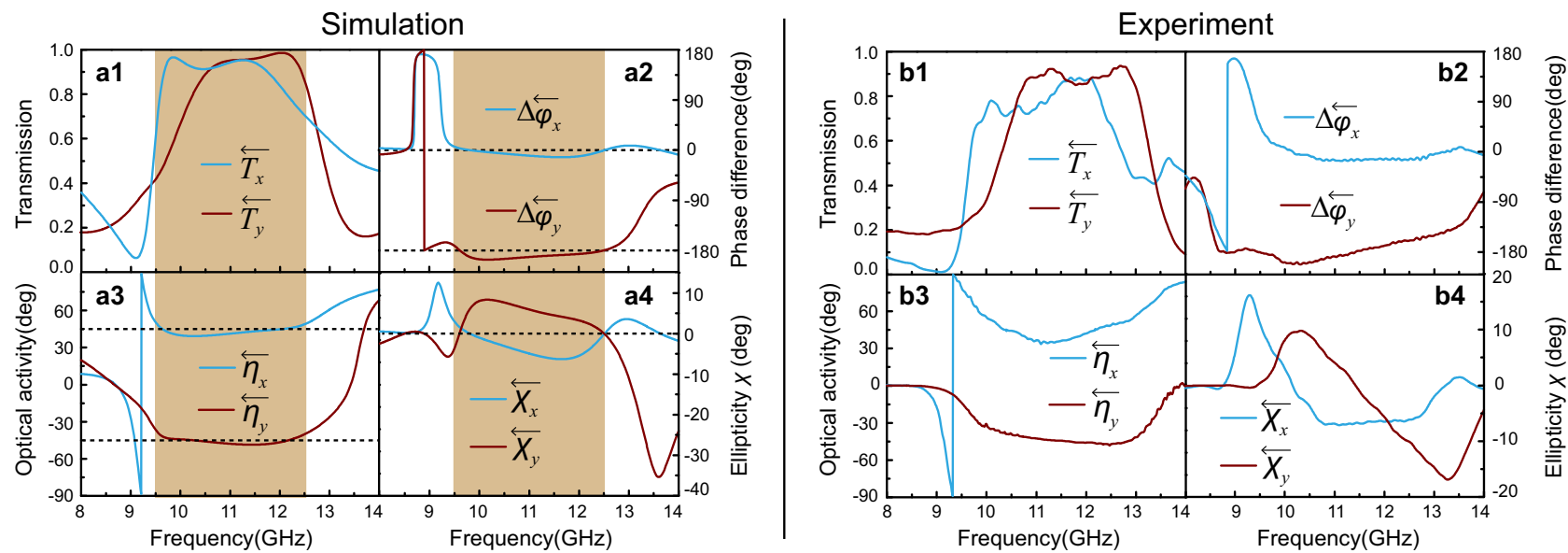

Fig. 5 a Simulated and $\mathbf{b}$ measured results of the FLCM for the backward propagating $x$ - and $y$-polarized waves. (a1 and $\mathbf{b} 1$ ) Transmission spectra, (a2 and b2) transmission phase differences, (a3 and b3) polarization azimuth rotation angles $\eta$, and (a4 and b4) calculated ellipticity angles $X$ of the transmitted waves

backward direction

$\Delta \overleftarrow{\varphi_{x}}=\overleftarrow{L t_{y x}}-\overleftarrow{L t_{x x}}=\check{L t_{x y}}-\overleftarrow{L t_{x x}}$

$\Delta \overleftarrow{\varphi_{y}}=\overleftarrow{L t_{y y}}-\overleftarrow{L t_{x y}}=\check{L t_{y y}}-\check{L t_{y x}}$

From Fig. 4a1-a4, the amplitudes of four components are almost equal to each other and the phase difference between $\overrightarrow{t_{x x}}$ and $\overrightarrow{t_{x y}}\left(\overrightarrow{t_{y x}}, \overrightarrow{t_{y y}}\right)$ is closed to $0^{\circ}\left(-90^{\circ},+90^{\circ}\right)$ in the offresonance frequency range, corresponding to the forward propagating matrix of Eq. (10) and indicating a $45^{\circ}$ polarization rotation for $x$ - and $y$-polarized incident waves in the backward direction. For the backward propagating $x$ - and $y$-polarized waves, the total transmissions $\left(\overleftarrow{T_{x}}=\left|\overrightarrow{t_{x x}}\right|^{2}+\left|\overrightarrow{t_{y x}}\right|^{2} \quad\right.$ and $\overleftarrow{T_{y}}=\left|\overrightarrow{t_{x y}}\right|^{2}+\left|\overrightarrow{t_{y y}}\right|^{2}$ ) are greater than 0.6 and 0.4 in the off- resonance frequency of $9.5-12.5 \mathrm{GHz}$, respectively, as shown in Fig. 5a1. Furthermore, the total transmissions can be abruptly improved by decreasing the off-resonance bandwidth. The transmission phase differences of $\Delta \overleftarrow{\varphi_{x}}$ and $\Delta \overleftarrow{\varphi_{y}}$ calculated by Eqs. (19) and (20) tend to be $0^{\circ}$ and $-180^{\circ}$ in the off-resonance frequency region (Fig. 5a2). To evaluate the optical activity of our proposed structure, the polarization azimuth rotation angle $\eta$ is defined as

$\eta=\frac{1}{2} \arctan \left(S_{2} / S_{1}\right)$

where $S_{1}$ and $S_{2}$ are taken from Stokes parameters. The polarization azimuth rotation angle $\eta$ ranges from $-90^{\circ}$ to $+90^{\circ}$. Figure $5 \mathrm{a} 3$ demonstrates the polarization azimuth rotation angle $\eta$ closed to $+45^{\circ}$ or $-45^{\circ}$ for $x$ - or $y$-polarized incident wave in the range of $9.5-12.5 \mathrm{GHz}$, respectively. Meanwhile, the ellipticity angle for $x$ - and $y$-polarized incident waves are greater than $-6.3^{\circ}$ 

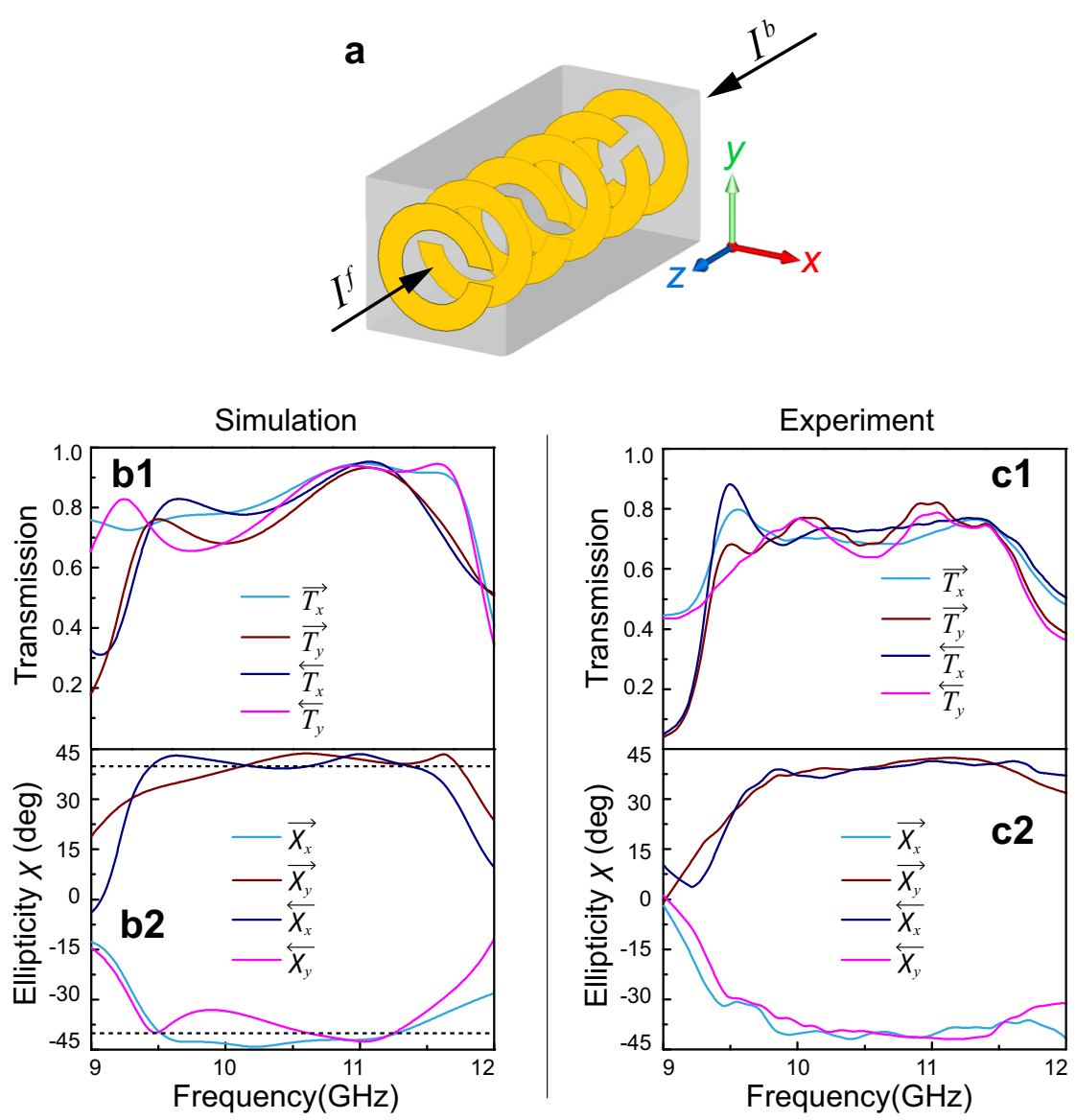

Fig. 6 Multilayer chiral metamaterial as an abnormal m-QWP. a Schematic of the unit cell. $\mathbf{b}$ Simulated and $\mathbf{c}$ measured results of transmission spectra (upper panels) and ellipticity angles (lower panels)

and lower than $8.3^{\circ}$ (Fig. 5a4), respectively, indicating a nearly linear polarization. Therefore, our proposed FLCM can realize a near $45^{\circ}$ polarization rotation with high transmission in broad bandwidth when the LP waves propagate backward. The experimental results shown in Fig. $5 b$ are consistent with the simulated ones very well.

The proposed FLCM not only has bifunctionality but also exhibits excellent properties of high transmission and broadband, which benefits from the formation of F-P-like interference (see Supplementary Information, Section II). Conventional method for tracking the F-P-like scattering processes is very complex due to the four layer interfaces, so we adopt a more convenient method of $4 \times 4$ transfer matrix in this case, ${ }^{42}$ where each layer of metasurfaces is treated as a zero-thickness impedance surface with the modified transmission and reflection coefficients acquired from numerical simulations. As shown in Fig. S1c, d, the calculated results of co- and cross-polarization transmission coefficients for $x$ - and $y$-polarized incident waves coincide with simulated results (Fig. 4a1, a3) very well.

Abnormal meta-quarter-wave plate $(\theta=\pi / 2)$

To better demonstrate the flexibility of design strategy, we cascade three m-QWPs to construct an abnormal m-QWP (Eq. (16)), as shown in Fig. 6a. The top two m-QWPs form an m-HWP and the back m-QWP is oriented at $45^{\circ}$ with respect to the $x$ axis, leading to a mirror symmetry breaking in the propagation direction. To balance the relationship between transmission amplitude and phase, we slightly increase the separation distance between two adjacent metasurfaces by $4.0 \mathrm{~mm}$ and keep other geometric parameters fixed. Figure $6 \mathrm{~b}, \mathrm{c}$ shows the simulated and experimental transmission results for the forward and backward propagating LP waves. Just as the theoretical analysis, the composite structure can convert the $x$ - or $y$-polarized incident wave into LCP or RCP waves with high transmission efficiencies in broadband for the forward propagation direction; while it reverses its polarization conversion function for the backward propagation, i.e., convert $x$ - or $y$-polarized incident wave into RCP or LCP waves, respectively.

\section{DISCUSSION}

In summary, utilizing the inverse design method based on the scattering matrix, we demonstrate two multifunctional chiral metamaterials with different polarization controls for oppositely propagating directions, theoretically and experimentally. The FLCM composed of two m-QWPs with a twist angle of $45^{\circ}$ is experimentally demonstrated to generate a nearly perfect LCP or RCP wave with a high transmission in a broad bandwidth, when the $x$ - or $y$-polarized incident wave propagates forward. On the contrary, the FLCM works as a near $45^{\circ}$ polarization rotation for the backward LP waves. We also verify that the high transmission at the off-resonance frequencies results from the F-P-like interference effect by the transfer matrix method. Furthermore, by employing such $\mathrm{m}$-QWP, we design a high-performance bifunctional $\mathrm{m}$-QWP that converts the forward (backward) propagating $x$ - or $y$-polarized wave into the LCP (RCP) or RCP (LCP) wave. The proposed chiral metamaterials show many merits, such as high transmission, broad bandwidth, and bifunctionality, and may find potential applications in polarization-controlled devices. Moreover, the design strategy can be extended to terahertz and optical frequencies, enabling extensive applications. 


\section{METHODS}

Simulation

Numerical simulations are calculated by using the commercial software CST Microwave Studio. Due to the periodic structure, a single unit cell is simulated with periodic boundary conditions and wave ports in $x, y$ and $z$ directions, respectively. The dielectric constant of F4B material is 2.1 with a loss tangent of 0.003 . The copper film is simulated with a thickness of $0.035 \mathrm{~mm}$ and a default conductivity of $5.99 \times 10^{7} \mathrm{~S} / \mathrm{m}$.

\section{Fabrication and measurement}

All the chiral metamaterial specimens are consisted of $30 \times 30$ unit cells and fabricated using the standard printed circuit board technology. The transmission measurements are carried out by a vector network analyzer (Agilent N5230) with two LP lens horn antennas in an anechoic chamber. The polarization state of the incidence can be adjusted by rotating the emitter with an angle of $0^{\circ}$ or $90^{\circ}$ to obtain either $x$ - or $y$-polarized $E M$ wave, and the receiver can be reconfigurable to co-polarization or crosspolarization states by rotating with an angle of $0^{\circ}$ or $90^{\circ}$ with respect to the emitting antenna, so that four components of the LP waves are all measured.

\section{DATA AVAILABILITY}

The data that support the findings of this study are available from the corresponding authors upon reasonable request.

\section{ACKNOWLEDGEMENTS}

This work was supported by grants from Beijing Municipal Science and Technology Project (Z191100004819002), the National Natural Science Foundation of China (Nos. 51741202, 51788104, 51532004, and 11704216), the National Youth Top-Notch Talent Support Program, the Fundamental Research Funds for the Central Universities, and the National Postdoctoral Program for Innovative Talents (BX201801148).

\section{AUTHOR CONTRIBUTIONS}

Y.B. and C.L. conceived the idea and designed the chiral metamaterials. C.L. performed the numerical simulations and experiment measurements. Q.Z., Y.Y., and H.C. assisted the experimental design and microwave measurements. J.Z. and L.Q. guided the theoretical analysis. All authors contributed to analysing the results. Y.B. and C.L. wrote the manuscript with revisions from other authors.

\section{ADDITIONAL INFORMATION}

Supplementary information accompanies the paper on the npj Computational Materials website (https://doi.org/10.1038/s41524-019-0230-z).

Competing interests: The authors declare no competing interests.

Publisher's note Springer Nature remains neutral with regard to jurisdictional claims in published maps and institutional affiliations.

\section{REFERENCES}

1. Agrawal, A. \& Choudhary, A. Perspective: Materials informatics and big data: Realization of the "fourth paradigm" of science in materials science. Apl. Mater. 4, 1-17 (2016).

2. Soukoulis, C. M. \& Wegener, M. Past achievements and future challenges in the development of three-dimensional photonic metamaterials. Nat. Photon. 5, 523-530 (2011).

3. Bi, K., Zhu, W., Lei, M. \& Zhou, J. Magnetically tunable wideband microwave filter using ferrite-based metamaterials. Appl. Phys. Lett. 106, 173507 (2015).

4. $\mathrm{Xu}, \mathrm{H}$. et al. Switchable complementary diamond-ring-shaped metasurface for radome application. IEEE Antennas Wirel. Propag. Lett. 17, 2494-2497 (2018).

5. Bi, K. et al. Experimental demonstration of ultra-large-scale terahertz all-dielectric metamaterials. Photon. Res. 7, 457-463 (2019).

6. Pendry, J. A chiral route to negative refraction. Science 306, 1353-1355 (2004).

7. Wang, B., Zhou, J., Koschny, T. \& Soukoulis, C. M. Nonplanar chiral metamaterials with negative index. Appl. Phys. Lett. 94, 151112 (2009).

8. Gansel, J. K. et al. Gold helix photonic metamaterial as broadband circular polarizer. Science 325, 1513-1515 (2009).
9. Zhao, Y., Belkin, M. A. \& Alù, A. Twisted optical metamaterials for planarized ultrathin broadband circular polarizers. Nat. Commun. 3, 870 (2012).

10. Cui, Y., Kang, L., Lan, S., Rodrigues, S. \& Cai, W. Giant chiral optical response from a twisted-arc metamaterial. Nano Lett. 14, 1021-1025 (2014).

11. Menzel, C. et al. Asymmetric transmission of linearly polarized light at optical metamaterials. Phys. Rev. Lett. 104, 253902 (2010).

12. Huang, C., Feng, Y., Zhao, J., Wang, Z. \& Jiang, T. Asymmetric electromagnetic wave transmission of linear polarization via polarization conversion through chiral metamaterial structures. Phys. Rev. B 85, 195131 (2012).

13. Kenanakis, G. et al. Three-dimensional infrared metamaterial with asymmetric transmission. ACS Photonics 2, 287-294 (2015).

14. Zhang, F. et al. All-dielectric metasurfaces for simultaneous giant circular asymmetric transmission and wavefront shaping based on asymmetric photonic spin-orbit interactions. Adv. Funct. Mater. 27, 1704295 (2017).

15. Wang, S. et al. Asymmetric transmission based on magnetic resonance coupling in 3D-printed metamaterials. Appl. Phys. Lett. 113, 081904 (2018).

16. Ye, Y. \& He, S. $90^{\circ}$ polarization rotator using a bilayered chiral metamaterial with giant optical activity. Appl. Phys. Lett. 96, 203501 (2010).

17. Cong, L. et al. Highly flexible broadband terahertz metamaterial quarter-wave plate. Laser Photon. Rev. 8, 626-632 (2014).

18. Kaschke, J. et al. A helical metamaterial for broadband circular polarization conversion. Adv. Opt. Mater. 3, 1411-1417 (2015).

19. Selvanayagam, M. \& Eleftheriades, G. V. Design and measurement of tensor impedance transmitarrays for chiral polarization control. IEEE Trans. Microw. Theory Tech. 64, 414-428 (2016).

20. Pfeiffer, C. \& Grbic, A. Bianisotropic metasurfaces for optimal polarization control: analysis and synthesis. Phys. Rev. Appl. 2, 044011 (2014).

21. Park, H. S. et al. A general recipe for nondispersive optical activity in bilayer chiral metamaterials. Adv. Opt. Mater. 7, 180 (1729).

22. Plum, E. \& Zheludev, N. I. Chiral mirrors. Appl. Phys. Lett. 106, 221901 (2015).

23. Kang, L. et al. Preserving spin states upon reflection: linear and nonlinear responses of a chiral meta-mirror. Nano Lett. 17, 7102-7109 (2017).

24. Wen, D. et al. Metasurface device with helicity-dependent functionality. Adv. Opt. Mater. 4, 321-327 (2015)

25. Forouzmand, A et al. Double split-loop resonators as building blocks of metasurfaces for light manipulation: bending, focusing, and flat-top generation. J. Opt. Soc. Am. B 33, 1411-1420 (2016).

26. Balthasar Mueller, J. P., Rubin, N. A., Devlin, R. C., Groever, B. \& Capasso, F. Metasurface polarization optics: independent phase control of arbitrary orthogonal states of polarization. Phys. Rev. Lett. 118, 113901 (2017)

27. Wang, Q. et al. Reflective chiral meta-holography: multiplexing holograms for circularly polarized waves. Light Sci. Appl. 7, 25 (2018).

28. Chen, W. T. et al. High-efficiency broadband meta-hologram with polarizationcontrolled dual images. Nano Lett. 14, 225-230 (2013).

29. Cai, T. et al. High-efficiency and full-space manipulation of electromagnetic wave fronts with metasurfaces. Phys. Rev. Appl. 8, 034033 (2017).

30. Tong, $C$. et al. High-performance bifunctional metasurfaces in transmission and reflection geometries. Adv. Opt. Mater. 5, 1600506 (2017).

31. Lei, Z. et al. Transmission-reflection-integrated multifunctional coding metasurface for full-space controls of electromagnetic waves. Adv. Funct. Mater. 28 1802205 (2018).

32. Cui, T. J., Qi, M. Q., Wan, X., Zhao, J. \& Cheng, Q. Coding metamaterials, digital metamaterials and programmable metamaterials. Light Sci. Appl. 3, e218 (2014).

33. Liu, L., Kang, L., Mayer, T. S. \& Werner, D. H. Hybrid metamaterials for electrically triggered multifunctional control. Nat. Commun. 7, 13236 (2016)

34. Chen, K. et al. A reconfigurable active huygens' metalens. Adv. Mater. 29, 1606422 (2017).

35. Zhang, S. et al. Photoinduced handedness switching in terahertz chiral metamolecules. Nat. Commun. 3, 942 (2012).

36. Zhou, J. et al. Terahertz chiral metamaterials with giant and dynamically tunable optical activity. Phys. Rev. B 86, 035448 (2012).

37. Kan, T. et al. Enantiomeric switching of chiral metamaterial for terahertz polarization modulation employing vertically deformable MEMS spirals. Nat. Commun. 6, 8422 (2015).

38. Yin, X. et al. Active chiral plasmonics. Nano Lett. 15, 4255-4260 (2015).

39. Chen, K. et al. Dynamic control of asymmetric electromagnetic wave transmission by active chiral metamaterial. Sci. Rep. 7, 42802 (2017).

40. Li, Z. et al. Simultaneous generation of high-efficiency broadband asymmetric anomalous refraction and reflection waves with few-layer anisotropic metasurface. Sci. Rep. 6, 35485 (2016).

41. Chen, Y., Gao, J. \& Yang, X. Direction-controlled bifunctional metasurface polarizers. Laser Photon. Rev. 12, 1800198 (2018).

42. Grady, N. K. et al. Terahertz metamaterials for linear polarization conversion and anomalous refraction. Science 340, 1304-1307 (2013). 
Open Access This article is licensed under a Creative Commons Attribution 4.0 International License, which permits use, sharing, adaptation, distribution and reproduction in any medium or format, as long as you give appropriate credit to the original author(s) and the source, provide a link to the Creative Commons license, and indicate if changes were made. The images or other third party material in this article are included in the article's Creative Commons license, unless indicated otherwise in a credit line to the material. If material is not included in the article's Creative Commons license and your intended use is not permitted by statutory regulation or exceeds the permitted use, you will need to obtain permission directly from the copyright holder. To view a copy of this license, visit http://creativecommons. org/licenses/by/4.0/.

(c) The Author(s) 2019 